\title{
Selbstwechselwirkung des elektromagnetischen Wellenfeldes gemäß einer aus einer projektiven Relativitätstheorie folgenden nichtlinearen Elektromagnetik
}

\author{
Von Ernst Schmutzer \\ Aus dem Theoretisch-Physikalischen Institut der Universität Jena \\ (Z. Naturforschg. 14 a, 489_492 [1959]; eingegangen am 19. Januar 1959)
}

\begin{abstract}
Im Rahmen einer in einer früheren Arbeit entwickelten nichtlinearen Elektrodynamik, die auf eine konvergente Feldenergie einer Punktsingularität führte, wird hier die Selbstwechselwirkung freier elektromagnetischer Felder untersucht. Es gelingt, Näherungslösungen des Feldgleichungssystems zu finden, die in erster Approximation der Maxwell-Theorie entsprechen. Darüberhinaus tritt ein gewisses raumzeitliches Schwanken von Feldgrößen interferierender Wellen auf, welches durch einen dimensionslosen Faktor charakterisiert wird, in den die Gravitationskonstante, Frequenz und Amplitude eingehen. Außerdem zeigt sich, daß ein starkes elektromagnetisches Feld gegenüber der Wellenausbreitung quasimetallisch wirkt. Ein eigentliches Streuphänomen wird für zwei entgegenlaufende Wellen nicht vorausgesagt.
\end{abstract}

\section{§ 1. Gleichungen des freien Feldes und andere Beziehungen}

Die Bezeichnungen für die Feldgrößen und die Koordinaten des Mrnkowski-Raumes übernehmen wir aus der bereits erwähnten Arbeit ${ }^{1}$ des Verf., die als zugehöriger erster Teil angesehen werden kann. Die Feldgleichungen des freien Feldes schreiben sich dann

$$
\begin{gathered}
\operatorname{rot} \mathfrak{S}=\frac{1}{c} \frac{\partial \mathfrak{D}}{\partial t}, \quad(1) \quad \operatorname{rot} \mathfrak{F}=-\frac{1}{c} \frac{\partial \mathfrak{B}}{\partial t}, \\
\operatorname{div} \mathfrak{D}=0, \quad(3) \quad \operatorname{div} \mathfrak{B}=0, \\
\square J=\frac{(2 x)^{3 / 2}}{2}(\mathfrak{S} \mathfrak{B}-(\mathfrak{D})
\end{gathered}
$$

( $x$ kosmische Gravitationskonstante),

während die Materialgleichungen des freien Feldes im benutzten Maßsystem lauten:

$$
\mathfrak{S}=\frac{J^{3}}{(2 \varkappa)^{3 / 2}} \mathfrak{B}, \quad(6) \quad \mathfrak{D}=\frac{J^{3}}{(2 \varkappa)^{3 / 2}} \mathfrak{F} .
$$

Damit kann man den Gln. (1) und (3) auch die Form geben:

$$
\begin{gathered}
\operatorname{rot} \mathfrak{B}=-3 \operatorname{grad} \ln J \times \mathfrak{B}+\frac{1}{c} \frac{\partial \mathfrak{E}}{\partial t}+\frac{3}{c} \mathfrak{F} \frac{\partial \ln J}{\partial t}, \\
\operatorname{div} \mathfrak{E}=-3 \mathfrak{g r a d} \ln J .
\end{gathered}
$$

Die in üblicher Weise durchgeführten Eliminationen führen auf die Wellengleichungen:

$$
\begin{aligned}
\square \mathcal{F} & =\frac{3}{c^{2}} \frac{\partial \mathfrak{F}}{\partial t} \frac{\partial \ln J}{\partial t}+\frac{3}{c^{2}} \mathfrak{F} \frac{\partial^{2} \ln J}{\partial t^{2}}-3(\mathfrak{F} \nabla) \operatorname{grad} \ln J \\
& -3(\operatorname{grad} \ln J \nabla)\left(E-\frac{3}{c} \operatorname{grad} \frac{\partial \ln J}{\partial t} \times \mathfrak{B}, \quad(10)\right.
\end{aligned}
$$

1 E. Schmutzer, Z. Naturforschg. 14 a, 486 [1959]; voranstehende Arbeit, dort weitere Literatur.

$$
\begin{aligned}
\square \mathfrak{B}=\frac{3}{c^{2}} & \frac{\partial \mathfrak{B}}{\partial t} \frac{\partial \ln J}{\partial t}+3(\mathfrak{B} \nabla) \operatorname{grad} \ln J \\
& -3(\operatorname{grad} \ln J \nabla) \mathfrak{B}-3 \mathfrak{B}(\Delta \ln J) \\
& -\frac{3}{c} \operatorname{grad} \frac{\partial \ln J}{\partial t} \times \mathfrak{F} .
\end{aligned}
$$

Aus Gl. (5) erkennt man, daß immer dann, wenn $\mathfrak{F} \mathfrak{D}=\mathfrak{S} \mathfrak{B}$ ist, $J=J_{0}$ als Lösung folgt, so daß in diesem Fall die Reduktion auf die Maxwellsche Theorie vorliegt. Insbesondere ist, wie sich zeigen wird, eine ebene Welle Lösung unseres Gleichungssystems.

In Viererschreibweise kann man die obigen Feld. gleichungen auch zusammenfassen durch das System

$$
\begin{gathered}
H_{i j}=A_{j, i}-A_{i, j}, \\
H_{i j, j}+\frac{3 J, j}{J} H_{i j}=0, \\
\square J=\frac{J^{3}}{4} H_{i j} H_{i j} .
\end{gathered}
$$

Um die Rechnungen zu vereinfachen, wollen wir generell von der LoRentz-Bedingung

Gebrauch machen.

$$
A_{j, j}=0
$$

Das neue $J$-Feld haben wir in früheren Arbeiten ${ }^{1}$ als neue physikalische Realität aufgefaßt. Die Dimension von $J$ entspricht einem reziproken Potential. Wir fragen jetzt deshalb, wann man die Formel

$$
J^{2}=-2 / A_{i} A_{i}
$$

als Lösung der Feldgleichungen ansehen kann. Wir werden dafür eine wichtige Bedingungsgleichung ableiten: 
Eliminiert man mit Hilfe von (16) das J-Feld aus (13), so resultiert:

$$
3 H_{i j} A_{s} A_{s, j} A_{i}+A_{i, j, j} A_{i} A_{s} A_{s}=0 .
$$

Die analoge Elimination des $J$-Feldes aus (14) ergibt:

$$
A_{j, i} A_{i, j} A_{s} A_{s}-3 A_{j, i} A_{l, j} A_{i} A_{l}=0 .
$$

Addiert man die beiden letzten Gleichungen, so findet man

$3 A_{i, j} A_{l, j} A_{i} A_{l}$

$-A_{i, j, j} A_{s} A_{s} A_{i}-A_{i, j} A_{j, i} A_{s} A_{s}=0$.

Eliminiert man daraus die zweiten Ableitungen der Potentiale vermöge (13), so kommt man zu der folgenden Bedingungsgleichung dafür, daß (16) Lösung des Gleichungssystems ist:

$$
3 A_{i} A_{l} A_{j, i} A_{l, j}=A_{s} A_{s} A_{i, j} A_{j, i} .
$$

Für die Elektrostatik und auch andere einfachere Fälle ist diese Bedingung erfüllt, d. h. $J$ kann gleich in der Gestalt (16) übernommen werden. Man kann dieses Ergebnis leicht für das Potential $\varphi$ einer Punktladung verifizieren, wo tatsächlich $J$ und $\varphi$ reziprok verknüpft sind ${ }^{1}$. Darüber hinaus erkennen wir aus (16) einiges bezüglich der physikalischen Bedeutung von $J$. $J$ ist nämlich in diesen Fällen rational verknüpft mit der in der Elektrodynamik merkwürdigen Invarianten $A_{i} A_{i}$.

\section{§ 2. Interferenz elektromagnetischer Wellen}

In diesem Abschnitt soll eine Näherungslösung des obigen nichtlinearen Gleichungssystems abgeleitet werden, die der Überlagerung mehrerer ebener Wellen entspricht. Für die BoRN-INFELd-Theorie hat dieses Problem SchröDinger ${ }^{2}$ näherungsweise gelöst, indem er die Elektromagnetik komplex formulierte. Für unsere Zwecke bringt diese Methode allerdings keine Vorteile, so daß wir im Reellen bleiben werden.

Da die MaxwelL-Theorie als erste Näherung gültig sein soll, so beginnen wir mit folgendem Superpositionsansatz:

$$
\begin{array}{r}
\mathfrak{F}^{(1)}=\sum_{\nu} \mathfrak{U}_{\nu} \Omega_{\nu}=\sum_{\nu} \mathfrak{U}_{\nu} \cos \left(\mathfrak{f}^{(v)} \mathfrak{r}-\omega_{\nu} t+\lambda_{\nu}\right) \\
=\sum_{\nu} \mathfrak{U}_{\nu} \cos \left(k_{j}^{(\nu)} x_{j}+\lambda_{\nu}\right) .
\end{array}
$$

Dabei ist:

$$
\mathfrak{f}^{(v)}=\frac{\omega_{v}}{v_{v}} \mathfrak{n}^{(v)}, \quad k_{4}{ }^{(\nu)}=\frac{i \omega_{v}}{c},
$$

2 E. SchrödingEr, Proc. Roy. Irish. Acad. 47, 77 [1942]. wobei $\mathfrak{n}^{(v)}$ die Wellennormalenvektoren, $v_{v}$ die Phasengeschwindigkeiten, $\mathfrak{A}_{v}$ konstante Amplituden und $\lambda_{v}$ Phasenkonstanten bedeuten. Es ist zweckmäßig, folgende Rechenformeln festzuhalten:

$$
\begin{aligned}
\text { a) } \operatorname{grad} \Omega_{\nu} & =-\sin \left(\mathfrak{f}^{(v)} \mathfrak{x}-\omega_{\nu} t+\lambda_{v}\right) \mathfrak{f}^{(v)}, \\
\text { b) } \quad \frac{\partial \Omega_{v}}{\partial t} & =\omega_{\nu} \sin \left(\mathfrak{f}^{(v)} \mathfrak{x}-\omega_{\nu} t+\lambda_{v}\right) .
\end{aligned}
$$

Gehen wir mit dem Ansatz (21) in (2) ein, so folgt durch zeitliche Integration:

$$
\mathfrak{B}^{(1)}=c \sum_{\nu} \frac{\Omega_{v}}{\omega_{v}}\left(\mathfrak{f}^{(v)} \times \mathfrak{U}_{v}\right) .
$$

Setzt man diesen Ausdruck in (4) ein, so ist diese Gleichung wegen $\mathfrak{f}^{(v)}\left(\mathfrak{f}^{(v)} \times \mathfrak{U}_{v}\right)=0$ von selbst erfüllt. Da in erster Näherung $\mathfrak{B}^{(1)}=\mathfrak{S}^{(1)}, \mathfrak{F}^{(1)}=\mathfrak{D}^{(1)}$ gilt, so folgt weiter aus (3) und (1):

$$
\mathfrak{U}_{\nu} \mathfrak{f}^{(\nu)}=0, \quad(25) \quad v_{\nu}=c .
$$

Diese Näherungslösungen verwenden wir jetzt für die rechte Seite von (5), so daß wir, da $J$ im MaxweLLschen Fall gemäß unserer früheren Festsetzung ${ }^{1}$ $\sqrt{2 \varkappa}$ wird, dafür die näherungsweise gültige PorssonGleichung

$$
\square J=\frac{(2 \varkappa)^{3 / 2}}{2}\left(\mathfrak{B}^{(1) 2}-\mathfrak{F}^{(1) 2}\right)
$$

erhalten.

Durch Einsetzen der obigen Ausdrücke entsteht aus (27):

$$
\square J=\frac{(2 \varkappa)^{3 / 2}}{2} \sum_{\nu, \mu} h_{\nu, \mu} \Omega_{\nu} \Omega_{\mu} .
$$

Dabei haben wir folgende Abkürzungen eingeführt:

$$
\begin{aligned}
h_{\nu \mu}=\frac{c^{2}}{\omega_{\nu} \omega_{\mu}}\{ & \left(\mathfrak{U}_{\nu} \mathfrak{U}_{\mu}\right)(\mathfrak{f}(v) \mathfrak{f}(\mu)) \\
& \left.-\left(\mathfrak{f}^{(\nu)} \mathfrak{U}_{\mu}\right)\left(\mathfrak{f}(\mu) \mathfrak{U}_{\nu}\right)-\frac{\mathfrak{U}_{\nu} \mathfrak{A}_{\mu} \omega_{\nu} \omega_{\mu}}{c^{2}}\right\} .
\end{aligned}
$$

Die für unsere Zwecke sinnvolle physikalische Lösung von (28) ist

$$
J=\text { const }(1-M) .
$$

$M$ ist dabei folgender Ausdruck:

$$
\begin{aligned}
M=\frac{\varkappa}{2} \sum_{\nu, \mu} h_{\nu \mu}\left[\frac{\cos \left\{\left(k_{i}(v)-k_{i}(\mu)\right) x_{i}+\lambda_{\nu}-\lambda_{\mu}\right\}}{\left(k_{i}(v)-k_{i}(\mu)\right)\left(k_{i}(v)-k_{i}(\mu)\right)}\right. \\
\left.+\frac{\cos \left\{\left(k_{i}(v)+k_{i}(\mu)\right) x_{i}+\lambda_{\nu}+\lambda_{\mu}\right\}}{\left(k_{i}(v)+k_{i}(\mu)\right)\left(k_{i}(v)+k_{i}(\mu)\right)}\right] .
\end{aligned}
$$

Mit dem nunmehr gewonnenen Näherungsausdruck für $J$ haben wir dann in die strengen Feldgleichungen (10) und (11) einzugehen: Setzen wir im weiteren voraus:

$$
|M| \ll 1
$$


so können wir schreiben:

$$
\ln J=\ln \text { const }-M \text {, }
$$

bzw.

$$
\begin{array}{r}
\operatorname{grad} \ln J=-\operatorname{grad} M, \\
\partial \ln J / \partial t=-\partial M / \partial t .
\end{array}
$$

Für eine einzelne freie Welle ergibt sich $h_{\nu \mu}=0$, also ist eine freie ebene Welle exakte Lösung unserer Gleichungen.

Bezüglich der Wechselwirkung zweier ebener Wellen unterscheiden wir die beiden Extremfälle: Sind beide Wellen gleichgerichtet:

so finden wir:

$$
\mathfrak{f}^{(1)} / \omega_{1}=\mathfrak{f}^{(2)} / \omega_{2},
$$

$$
h_{\nu \mu}=0,
$$

d. h. in diesem Fall tritt kein Effekt auf. Sind dagegen beide Wellen entgegengesetzt gerichtet:

$$
\mathfrak{f}^{(1)} / \omega_{1}=-\mathfrak{f}^{(2)} / \omega_{2},
$$

so resultiert:

$$
h_{11}=0, h_{22}=0, h_{12}=h_{21}=-2 \mathfrak{U}_{1} \mathfrak{U}_{2} .
$$

Somit ergibt sich für $M$ :

$$
M=-2 \varkappa \mathfrak{U}_{1} \mathfrak{U}_{\mathbf{2}}\left[\frac{\cos \left\{\left(k_{i}(1)+k_{i}(2)\right) x_{i}+\lambda_{1}+\lambda_{2}\right\}}{\left(k_{i}(1)+k_{i}(2)\right)\left(k_{i}(1)+k_{i}(2)\right)}+\frac{\cos \left\{\left(k_{i}(1)-k_{i}(2)\right) x_{i}+\lambda_{1}-\lambda_{2}\right\}}{\left(k_{i}(1)-k_{i}(2)\right)\left(k_{i}(1)-k_{i}(2)\right)}\right] .
$$

Im Falle gleicher Frequenzen und der Phasenkonstanten $\lambda_{1}=\lambda_{2}=0$ folgt daraus $(\mathfrak{f}(1)=\mathfrak{f})$ :

$$
M=-\frac{\varkappa c^{2}}{\omega^{2}}\left(\mathfrak{U}_{1} \mathfrak{U}_{2}\right) \sin (\omega t-\mathfrak{l} \mathfrak{r}) \sin (\omega t+\mathfrak{H} \mathfrak{r}) \text {. }
$$

Stehen beide F-Felder aufeinander senkrecht, so verschwindet auch in diesem Fall der Effekt.

Bemerkenswert ist in diesem Zusammenhang, daß die Größe des Effektes bestimmt wird durch die dimensionslose Konstante:

$$
\sigma=\frac{\varkappa c^{2}}{\omega^{2}}\left(\mathfrak{A}_{1} \mathfrak{A}_{2}\right) \approx 10^{-27} \frac{\left(\mathfrak{U}_{1} \mathfrak{U}_{2}\right)}{\omega^{2}}
$$

(im absoluten Maßsystem).

Überraschend ist dabei, daß der Effekt mit abnehmender Frequenz zunimmt und daß, falls die Verhältnisse bei höheren Näherungen nicht grundsätzlich modifiziert werden, ein kritisches AmplitudenFrequenz-Verhältnis existiert, für welches $\sigma=1$ wird, wodurch Umkehr der Richtungen der Vektoren (5) und $\mathfrak{D}$, resp. $\mathfrak{S}$ und $\mathfrak{B}$ eintritt.

Für $(34$ a) und $(34$ b) finden wir in diesem vereinfachten Fall entgegenlaufender Wellen, wenn wir für die weiteren Betrachtungen im $\sigma \quad \mathfrak{U}_{1}=\mathfrak{U}_{2}=\mathfrak{U}$ gesetzt denken:

$$
\begin{aligned}
\operatorname{grad} \ln J & =-\sigma \mathfrak{f} \sin (2 \mathfrak{f} \mathrm{r}), \\
\frac{\partial \ln J}{\partial t} & =\sigma \omega \sin (2 \omega t) .
\end{aligned}
$$

Damit nehmen die Feldgleichungen (10) und (11) die Form an:

$$
\begin{aligned}
\square \mathfrak{F}= & \frac{3 \sigma \omega}{c^{2}} \sin (2 \omega t) \frac{\partial \mathfrak{F}}{\partial t}+\frac{6 \sigma \omega^{2}}{c^{2}} \cos (2 \omega t) \mathfrak{F} \\
& +3 \sigma \sin (2 \mathfrak{f})(\mathfrak{f} \nabla) \mathfrak{F},
\end{aligned}
$$

$$
\begin{aligned}
\square \mathfrak{B}= & \frac{3 \sigma \omega}{c^{2}} \sin (2 \omega t) \frac{\partial \mathfrak{B}}{\partial t}+\frac{6 \sigma \omega^{2}}{c^{2}} \cos (2 \omega t) \mathfrak{B} \\
& +3 \sigma \sin (2 \mathfrak{f})(\mathfrak{f} \nabla) \mathfrak{B} .
\end{aligned}
$$

Das E-Feld und $\mathfrak{B}$-Feld pflanzen sich also nach demselben Gesetz fort. Außerdem sind die Differentialgleichungen linear in $\mathfrak{F}$ und $\mathfrak{B}$. Wir gehen in (43) mit dem folgenden Separationsansatz

$$
\mathfrak{E}=\mathbb{S}(\mathfrak{r}) T(t)
$$

ein und erhalten:

$$
\begin{aligned}
& \triangle \text { (S) }-3 \sigma \sin (2 \mathfrak{f} \mathfrak{x})(\mathfrak{f} \nabla) \text { Ś }+a^{2} \text { (S) }=0, \\
& T^{\prime \prime}+3 \sigma \omega \sin (2 \omega t) T^{\prime} \\
& +6 \sigma \omega^{2} \cos (2 \omega t) T+a^{2} c^{2} T=0 .
\end{aligned}
$$

Die letzte Gleichung lösen wir approximativ mit dem Ansatz:

$$
T=A+B \cos (\omega t)+\ldots
$$

und finden durch Koeffizientenvergleich:

$$
\begin{gathered}
A=0, \quad B=\text { beliebig, } \\
a^{2}=\frac{\omega^{2}}{c^{2}}\left(1-\frac{3}{2} \sigma\right) .
\end{gathered}
$$

Unsere nächsten Untersuchungen gelten jetzt dem räumlichen Feldanteil: Für (S) beginnen wir mit dem Ansatz:

$$
\text { (S) }=2 \mathfrak{U} \cos (\mathfrak{f} r) \text {. }
$$

Durch Einsetzen in (46) überzeugt man sich leicht, daß durch (51) bereits approximativ die Differentialgleichung befriedigt wird, wenn man wählt:

$$
\mathfrak{k}^{2}=a^{2}\left(1-\frac{3 \sigma}{2}\right)^{-1} \text {. }
$$

Eliminiert man $a^{2}$ aus (52) und (50), so folgt:

$$
v=c \text {. }
$$


Die Gesamtlösung können wir damit schreiben:

$$
\mathfrak{F}=2 \mathfrak{A} \cos (\mathfrak{f} \mathfrak{r})[\cos (\omega t)+\ldots] .
$$

Betrachtet man nun die früher abgeleitete Energiebilanz ${ }^{1}$ [Gl. (24)], so sieht man, daß wegen der Zeitabhängigkeit von $J$ ein Energieerhaltungssatz im eigentlichen Sinne streng nicht mehr gilt. Jedoch ist der Effekt nahezu verschwindend klein.

\section{§ 3. Wellenausbreitung in einem homogenen elektrostatisch-magnetostatischen Feld}

Der Einfachheit halber betrachten wir eine einzige ebene Welle, deren Amplitude im Vergleich zum angelegten homogenen elektrostatischen Feld $\mathfrak{F}_{0}$ und homogenen magnetostatischen Feld $\mathfrak{B}_{0}$ vernachlässigbar sein soll. Näherungsweise entsteht also aus (5) :

$$
\square J=\frac{(2 \varkappa)^{3 / 2}}{2}\left(\mathfrak{B}_{0}^{2}-\left(\mathfrak{F}_{0}^{2}\right)\right. \text {. }
$$

Wir vermuten als sinnvolle physikalische Lösung:

$$
J=\text { const }\left[1+\frac{\varkappa}{6}\left(\mathfrak{B}_{0}^{2}-\left(\mathfrak{F}_{0}^{2}\right) \mathfrak{r}^{2}\right],\right.
$$

woraus

$$
\operatorname{grad} \ln J \approx \frac{\varkappa}{3}\left(\mathfrak{B}_{0}^{2}-\mathfrak{F}_{0}^{2}\right) \mathfrak{r}
$$

folgt. Die Wellengleichungen (10) und (11) nehmen damit die Form an:

$\square \mathfrak{F}+\varkappa\left(\mathfrak{B}_{0}{ }^{2}-\mathfrak{F}_{0}{ }^{2}\right)(\mathfrak{r} \nabla) \mathfrak{F}=-\varkappa \mathfrak{F}_{0}\left(\mathfrak{B}_{0}{ }^{2}-\mathfrak{F}_{0}^{2}\right)$,

$\square \mathfrak{B}+\varkappa\left(\mathfrak{B}_{0}^{2}-\left(\mathfrak{F}_{0}^{2}\right)(\mathfrak{r} \nabla) \mathfrak{B}=-2 \varkappa \mathfrak{B}_{0}\left(\mathfrak{B}_{0}^{2}-\mathfrak{F}_{0}^{2}\right)\right.$.

Die homogene Gleichung zu (58) wird näherungsweise befriedigt durch den Ansatz:

$$
\mathfrak{F}=\mathfrak{F}_{0}+\mathfrak{U} e^{-i(\omega t-[w / v] \mathfrak{n} \mathfrak{r})},
$$

der zu folgendem approximativen komplexen Brechungsindex führt:

$$
n=\frac{c}{v}=\sqrt{1+\frac{i \varkappa c^{2}}{\omega^{2}}(\mathfrak{r} \mathfrak{f})\left(\mathfrak{B}_{0}^{2}-\left(\mathfrak{F}_{0}^{2}\right) .\right.}
$$

Das angelegte statische Feld wirkt sich also aus wie ein Quasimetall, absorbierend nach dem Gesetz $\exp \left\{-\varkappa\left(\mathfrak{B}_{0}^{2}-\mathfrak{F}_{0}^{2}\right) x^{2}\right\}$. Je nach Vorzeichen von $\left(\mathfrak{B}_{0}^{2}-\mathfrak{G}_{0}^{2}\right)$ tritt Absorption oder Verstärkung ein. Eine Näherungslösung der inhomogenen Gleichung $\mathrm{zu}$ (58) ist für kleine $|\mathfrak{r}|$ :

$$
\mathfrak{F}=-\frac{\varkappa}{6}\left(\mathfrak{F}_{0}^{2}-\mathfrak{F}_{0}^{2}\right) \mathfrak{F}_{0} \mathrm{r}^{2},
$$

so daß als Gesamtlösung entsteht:

$$
\mathfrak{F}=\mathfrak{F}_{0}\left[1-\frac{\varkappa}{6}\left(\mathfrak{B}_{0}^{2}-\mathfrak{F}_{0}^{2}\right) \mathfrak{r}^{2}\right]+\mathfrak{U} e^{-i(\omega t-[w / v] \mathfrak{n} \mathfrak{r})} .
$$

Sehen wir uns nun (59) an, so ist diese Gleichung dieselbe wie die eben gelöste, so daß wir sofort schreiben können:

$$
\mathfrak{B}=\mathfrak{B}_{0}\left[1-\frac{\varkappa}{3}\left(\mathfrak{B}_{0}^{2}-\mathfrak{F}_{0}{ }^{2}\right) \mathfrak{r}^{2}\right]+\mathfrak{C} e^{-i(\omega t-[w / v] \mathrm{n} \mathfrak{r})} .
$$

Aus (9) und (2) resultiert für kleine $|\mathfrak{r}|$ :

$$
(\mathfrak{U} \mathfrak{\mathfrak { f }})=0, \quad \mathfrak{C}=\frac{c}{w} \mathfrak{f} \times \mathfrak{U}
$$

wie in der Maxwellschen Theorie. Auch Gl. (8) ist näherungsweise befriedigt. Setzt man die oben gefundenen Lösungen der Feldgleichungen in die Formel für den Poynting-Vektor ein, so sieht man, daß zwar der Betrag der Energieströmung im obigen Sinne modifiziert wird, daß aber eine eigentliche Streuung der Lichtwelle nicht resultiert.

Herrn Dr. PAul gilt mein aufrichtiger Dank für zahlreiche wertvolle Diskussionen. 